\title{
DIE BEIDEN DEUTSCHEN STAATEN IN DEN VEREINTEN NATIONEN
}

\author{
Doç. Dr. Ayşe Füsun ARSAVA
}

\section{Einleitung}

24 Jahre nach der Konstituierung zweier deutscher Staaten wurden die Deutsche Demokratische Republik und die Bundesrepublik Deutschland am 18. September 1973 als 134. und 135. Mitglied in die Vereinten Nationen aufgenommen. Die Aufnahme mittels Akklamation durch die Vollversammlung der Vereinten Nationen, nachdem der Sicherheitsrat dieser zuvor einstimmig die Aufnahme der beiden deutschen Staaten empfohlen hatte, markiert sowohl Ende wie Anfang. War es für die DDR Zeichen des endgültigen Durchbruchs aus der internationalen Isolation, so war es für die Bundesrepublik sichtbarer Ausdruck der Bereitschaft, ihrer wachsenden weltpolitischen Rolle und Verantwortung auch durch ihre Mitarbeit als Vollmitglied der Vereinten Nationen gerecht zu werden. Dieses Datum ist jedoch sicherlich nicht mit dem Beginn einer aktiven Politik beider Staaten in den Vereinten Nationen gleichzusetzen und dies vor allem dann nicht, wenn man die Sonderorganisationen mit in die Analyse einbezieht.

Im folgenden sollen die politischen, staats- und völkerrechtlichen Aspekte aufgeführt und diskutiert werden, die die Aufnahme der beiden deutschen Staaten in die Vereinten Nationen bis 1973 blockierten sowie diejenigen, die dann gerade diese Aufnahme ermöglichten. Daran anschließend soll die bisherige Politik von Bundesrepublik und DDR in den Vereinten Nationen kurz dargestellt werden.

II. Allein nein, zusammen ja-der Weg der beiden deutschen Staaten in die Vereinten Nationen

1. Voraussetzungen einer Mitgliedschaft in den Vereinten Nationen

Art. 4 der Charta der Vereinten Nationen legt die Bestimmungen zur Aufnahme in die Weltorganisation fest: 
(1) Mitglied der Vereinten Nationen können alle sonstigen friedliebenden werden, welche die Verpflichtungen aus dieser Charta übernehmen und nach dem Urteil der Organisation fähig und willens sind, diese Verpflichtungen zu erfüllen.

(2) Die Aufnahme eines solchen Staates als Mitglied der Vereinten Nationen erfolgt auf Empfehlung des Sicherheitsrats durch Beschlu $\beta$ der Generalversammlung.

Art. 4 legt somit sowohl rechtliche wie politische Konditionen fest. Der rechtliche Charakter ist vor allem durch die Notwendigkeit der Staatsqualität gegeben, der politische ist jedoch nicht minder wichtig. Ist die inhaltliche Auffüllung des Adjektivs "friedliebend" offen für politische und ideologische Positionen, so ist dennoch vor allem Art. 4,2 maßgebend für die politische Durchsetzbarkeit. Der Aufnahme eines neuen Mitglieds müssen neun der 15 Mitglieder des Sicherheitsrates zustimmen, wobei die Stimmen der fünf ständigen Mitglieder notwendig sind (Art. 27,3 Charta der Vereinten Nationen).

2. Die Behandlung der deutschen Frage bis Ende der 60er Jahre und ihre Implikationen für einen Beitritt in die Vereinten Nationen

\section{a) Die weltpoliuische Lage}

Seit der Gründung der beiden deutschen Staaten im Jahre 1949, die nicht zuletzt Ergebnis und Ausdruck des sich verschärfenden Ost-WestGegensatzes waren, ist die deutsche Frage, d.h. die Frage der friedlichen Wiedervereinigung und der Beziehungen der beiden deutschen Staaten zueinander, abhängig von der internationalen "Großwetterlage" und dabei insbesondere des bilateralen Beziehungsgefüges der beiden Supermächte USA und UdSSR. Beide sind neben Frankreich, der Volksrepublik China und dem Königreich Großbritannien und Nordirland ständige Mitglieder des Sicherheitsrates. Das Veto eines dieser fünf Staaten genügt, um die Aufnahme als Mitglied in die Vereinten Nationen zu verhindern.

Die Einbindung der beiden deutschen Staaten in die jeweiligen Bündnissysteme des Westens und Ostens (NATO und Europäische Gemeinschaft einerseits und Warschauer Pakt und Rat für Gegenseitige Wirtschaftshilfe andererseits) in den 50er Jahren, dokumentierte bereits früh politische Abhängigkeiten. Hinzu kam und kommt, da $\beta$ eine Lösung der deutschen Frage die Rechte und Verantwortlichkeiten der Vier Mächte unmittelbar berührt. Die deutschlandpolitischen Positionen beider deut- 
schen Staaten konnten so von Anfang an nur durchgesetzt werden, wenn die bündnispolitischen Partner-allen voran USA und UdSSR-ihnen machtpolitische Durcsetzungskraft verliehen.

Nun war es seit Gründung von Bundesrepublik und DDR dar erklärte Ziel aller Bundesregierungen bis 1969, der DDR die Anerkennung als Staat und damit auch die internationale Anerkennung zu versagen - eine Politik, die die DDR entschieden bekämpfte. Die von den beiden deutschen Staaten seit ihrer Gründung vertretenen Positionen zur Rechtslage Deutschlands waren Ausdruck dieser gegensätzlichen Standpunkte. Die Literatur zu diesem Thema füllt inzwischen Regale. Im folgenden sollen nur die wichtigsten für unsere Fragestellung relevanten staats-und völkerrechtlichen Positionen zusammenfassend dargestellt werden, ohne dabei alle Gesichtspunkte umfassend und detailliert aufzuführen. ${ }^{.}$

\section{b) Die Staatlichkeit der DDR}

Nach der in der Völkerrechtslehre überwiegend vertretenen Meinung sind zur Qualifikation als Staat drei Elemente Bedingung: Staatsgebiet, Staatsvolk und Staatsgewalt. Ferner ist es nach herrschender Rechtslehre notwendig, da $\beta$ sich die Staatgsgewalt gegenüber dem Staatsvolk durchgesetzt hat (Effektivität der Staatsgewalt) und eine gewisse Dauerhaftigkeit erwarten läßst. ${ }^{2}$

Gemeinsam war allen politischen und rechtlichen Argumentationen der Bundesregierungen bis 1969, da $\beta$ sie der DDR die Staatlichkeit absprachen. Nach der offiziellen Auffassung der Bundesrepublik, die von den Westmächien geteilt wird, ist das Deutsche Reich weder 1945 noch 1949, dem Gründungsjahr der beiden deutschen Staaten, untergegangen. Es besitzt, obgleich augenblicklich nicht organisiert und ohne politische Handlungsfähigkeit,' Rechtscharakter. Bis Ende der 60er Jahre war die These von der Identität der Bundesrepublik mit dem deutschen Reich in der westlichen Völkerrechtsliteratur - und nach offizieller Lesart vorherrschend. Allein die Bundesrepublik habe die demokratische Legiti-

$\bar{I}$ Im besonderen soll hier auf einen ekzessiven Anmerkungsapparat verzichtet werden. In den hier zitierten Werken ist eine Fülle weiterführender oder kontroverser Literatur angeführt.

2 Siehe dazu Johann G. Gleich, Die Anerkennung der DDR durch die Bundesrepublik. Eine völkerrechtliche Untersuchung nach dem Abschlu 3 des Vertrages über die Grundlagen der Beziehungen zwischen der Bundesrepublik Deutschland und der Deutschen Demokratischen Republik vom 21. Dezember 1972, dem gemeinsamen Beitritt zur Charta der Vereinten Nationen und dem Austausch Ständiger Vertretungen. Bern und Frankfurt/M. 1975 (Augsburger Schriften zum Staatsund Völkerrecht; 3), S. 25. 
mation für alle Deutschen zu sprechen, da sie-im Gegensatz zur DDR aus allgemeinen und freien Wahlen hervorgegangen sei.

Zwar übte die ostdeutsche Regierung auf ihrem Staatsgebiet effektive Staatsgewalt aus, aber diese Gewalt galt infolge der fehlenden demokratíschen Legitimation als unrechtmäßig und infolge der bedingungslosen Abhängigkeit von der sowjetischen Besatzungsmacht als nicht souverän. Das größ3te Defizit des DDRRegimes aber bestand darin, da $\beta$ es seiner Staatsgewalt bis zum Bau der Berliner Mauer nicht gelungen war, sein Staatsvolk von der Abwanderung abzuhalten; eine Gewalt, der das Volk davonlief, entbehrte nach westdeutscher Auffassung der Qualität eines Staats. ${ }^{3}$

Diese Auffassung wurcie letztmals von einer Bundesregierung (unter Bundeskanzler Kiesinger) bei der Aufnahme diplomatischer Beziehungen mit Rumänien am 1.2.1967 vertreten. ${ }^{4}$ Der Alleinvertretungsanspruch der Bundesrepublik Deutschland konnte in den 50er und 60er Jahren mit Hilie der westlichen Verbündeten erfolgreich verfolgt werden. Das Mitte] dazu war die Nichtanerkennungspolitik, die sich in der sog. HallsteinDoktrin am augenfällingsten manifestierte. In daieser wurde eine völkerrechtliche Anerkennung der DDR als "unfreundlicher Akt" gegenüber der Bundesrepublik definiert, der wiedrum den Abbruch der diplomatische Beziehungen mit der Bundesrepublik zur Folge hatte. Bis Anfang 196.) konnte so die DDR ledighich mit 13 Staaten, die alle kommunistisch regiert waren, diplomatische Beziehungen aufnehmen. ${ }^{5}$ Die internationale Anerkennung im Weltmaßstab blieb ihr verwehrt.

Die DDR hat sich stets gegen diesen Alleinvertretungsanspruch der Bundesrepublik ausgesprochen; ja zunächst hatte man diesen Alleinvertretungsanspruch für den eigenen Staat in Anspruch genommen. Die Verfassung der DDR von 1949 ist bester Beleg für die These, da $\beta$ man zunächst von dem Fortbestand Deutschlands ausging und die Rechtsnachfolge für diesen deutschen Staat beanspruchte. ${ }^{6}$ Seit 1951 ist man sukzessive von diesem.Rech.tsstandpunkt abgegangen. Jens Hacker unterscheidet in seinem umfassendē Werk 'Der Rechtsstatus Deutschlands

${ }^{3}$ Heinrich End, Zweimal deutsche Außenpolitik. Internationale Dimensionen des innerdeutschen Konflikts 1949-1972. Köln 1973, S. 24.

4 Siehe Europa Archiv, 1967, S. D 115.

5 DDR Handbuch. Hrsgg. voin Bundesministerium für innerdeutsche Beziehungen. Wissenschaftliche Leitung: Peter Christian Ludz unter Mitwirkung von Johannes Kuppe, 2. völlig überarb. und erw. Auflage. Köln 1979, S 286.

6 Gesetzblatt der DDR, Nr. 1 v. 8. Oktober 1949, Präambel, Art, 1, 1, 2, 4, Art. 25, 12 und Art. 118, 1. 
aus der Sicht der DDR"? die Jahre von 1951 bis 1955, in der die DDR die These von der Diskontinuität des deutschen Staates bei Verneinung der Teilrechtsnachfolge des Deutschen Reiches durch die DDR vertrat und die Zeit seit 1956. Seither gilt die offizielle Rechtsauffassung, da $\beta$ die DDR und die Bundesrepublik Deutschland Nachfolgestaaten des untergegangenen Deutschen Reiches sind (Dismembrationstheorie) ${ }^{8}$

\section{Die Politik der Bundesrepublik und der DDR in den 50er und 60er Jahren in den Vereinten Nationen}

$\mathrm{Da}$ die Deutschlandpolitik der Bundesrepublik von den Westmäcten und die der DDR von den kommunistisch regierten Staaten unterstützt wurde, war sowohl ein Veto der Westmächte gegen einen Eintritt der DDR in die Weltorganisation als auch ein Veto der UdSSR gegen einen Eintritt der Bundesrepublik unvermeidlich und kalkulierbar. Deshalb hatte die Bundesrepublik bis 1972 keinen Aufnahmeauftrag gestellt. Im Gegensatz dazu hattc die DDR 1966 einen Vorsto $\beta$ unternommen, der aber wie erwartet negativ beschieden wurde. Es kann mit Sicherheit davon ausgegangen werden, da $\beta$ es der DDR 1966 auch nicht um die Aufnahme selbst, als vielmehr um die Notifizierung eines Anspruches, um eine internationale Diskussion über den Status der DDR ging. Mit der Formulierung des Maximalziels "Aufnahme in die Vereinten Nationen" wurde eventuell auch die Erreichung eines Minimalziels erhofft: die Zuerkennung eines affiziellen Beobachterstatus und eine Formalisierung der Beziehungen zur Weltorganisation. ${ }^{9}$

Auch 1966 war der DDR jedoch kein Erfolg beschert. In den Jahren vorher waren alle Versuche gescheitert, im System der Vereinten Nationen "gleichberechtigt" mit der Bundesrepublik mitzuarbeiten. Bemühungen, in Sonderorganisationen der Vereinten Nationen (z.B. IAO, ITU, UPU, WHO) ${ }^{10}$ einzutreten, waren ebensowenig von Erfolg gekrönt wie die Teilnahme an internationalen Konferenzen.

7 Köln 1974 (Abhandlungen zum Ostrecht; 13).

${ }^{8} \mathrm{Zu}$ den verschiedenen Theorien zur Rechtslage Deutschlands und dabei auch zur Dismembration siehe Gleich (Anm. 2), S. 63-87.

- Siehe dazu Wilhelm Bruns, Die UNO-Politik der DDR. Stuttgart 1978 (Bonn aktuell; 41), S. 30.

10 Z.B. Peter Pawelk, Die UNO und das Deutschlandproblem. Das Deutschlandproblem im Spannungsfeld zwischen der Bundesrepublik Deutschland und den Vereinten Nationen-unter besonderer Berücksichtigung der AuPenpolitik der Bundesrepublik Deutschland-1949 bis 1967. Tübingen 1971 (Tübinger Studien zui Geschichte und Politik; 28), S. 114-119. Wilhelm Bruns, "Politik der selektiven Mitgliedschaft. Das Verhältnis der DDR zu den UN-Sonderorganisationen, insbesondere zur UNESCO," in: Vereinte Nationen, 1978, H. 5, S. 154-159, S. 154. 
Damit blieb ihr der Erfolg verwehrt, der schon früh zur formalisierten Mitarbeit der Bundesrepublik Deutschland im System der Vereinten Nationen geführt hatte. Bereits im November 1950 wurde sie in die FAO aufgenommen. Eine Mitgliedschaft folgte der anderen. "Praktisch kann man sagen, da $\beta$ die Bundesrepublik bis 1955 allen existierenden Sonderorganisationen und Konferenzen der Vereinten Nationen beigetreten. war, sich an der Wirtschaftskommission für Europa beteiligte und zu den meisten Hilfswerken der Vereinten Nationen Beiträge leistete." Darüber hinaus hatte die Bundesrepublik seit 1952 einen ständigen Beobachter beim Sitz der Vereinten Nationen in New York und seit 1953 in Genf. ${ }^{12}$

War die Bundesrepublik somit bereits seit den 50er Jahren aktiv im System der Vereinten Nationen tätig, so war die Mitarbeit der DDR auf nichtformalisierte Aktivitäten beschränkt. Zur Verbreitung der Stellungnahmen zu Tagesordnungspunkten der Vereinten Nationen-im Vordergrund standen dabei stets Fragen der Abrüstung und der Entkolonialisierung-war sie auf die Dienste ihrer verbündeten Staaten angewiesen. Hauptadressaten ihrer Stellungnahmen waren, UNESCO, der Sicherheitsrat und die Generalversammlung der Vereinten Nationen. Zeitlich gesehen versuchte die DDR in den 50er Jahren zunächst der Bundesrepublik in die Sonderorganisationen zu folgen und begann erst in den 60er Jahren die Bemühungen auf eine Mitgliedschaft in den Vereinten Nationen selbst zu konzentrieren.

\section{Die Regelung der innerdeutschen Beziehungen als Voraussetzung einer gemeinsamen Aufnahme in die Vereinten Nationen}

Ende der 60er Jahre hatte sich die weltpolitische Situation entscheidend geändert: das Klima der Konfrontation, das die 50er Jahre beherschte, war der Bereitschaft zur Kooperation gewichen. Je länger die DDR existierte, desto schwieriger wurde es für die Bundesrepublik, ihren Alleinvertretungsanspruch aufrechtzuerhalten. Konnte die Bundesrepublik bisher die Anerkennung der DDR durch andere nichtkommunistiche Staaten und ihre Aufnahme in die Vereinten Nationen prinzipiell verhindern, so hatte sich das Ende der 60er Jahre geändert. So wurden z.B. die Aufnahmeanträge der DDR in die WHO 1968 und in die UNESCO 1968 und 1970 nicht mehr uneingeschränkt, sondern nur noch temporär abgelehnt. Der internationale Druck auf die Bundesrepublik-sowohl im

"Ernst-Otto Czempiel, Macht und Kompromiß. Die Beziehungen der BRD zu den Vereinten Nationen 1958-1970. Düsseldorf 1971, S. 42.

12 Zum Status des ständigen Beobachters siehe Ernst-Otto Cempiel, "Die Vereinten Nationen und die Nichtmitglieder. Über die Praxis und die Möglichkeiten der internationalen Organisation," in: Politische Vierteljahresschrift, 1968, S. 56-81. 
Hinblick auf eine Neudefinition ihrer weltpolitischen Rolle als auch im Hinblick auf ihre Ost - und Deutschlandpolitik wurde zunehmend stärker. Aber nicht nur die internationale Lage, auch das politische Meinungsbild innerhalb der Bundesrepublik selbst hatte sich gewandelt und ermöglichte eine Neuformulierung der Ost- und Deutschlandpolitik sowie ihre politische Durchsetzung.

"Eine völkerrechtliche Anerkennung der DDR durch die Bundesregierung kann nicht in Betracht kommen. Auch wenn zwei Staaten in Deutschland existieren, sind sie doch füreinander nicht Ausland; ihre Beziehungen zueinander können nur von besonderer Art sein."13 Mit dieser Aaussage in der Regierungserklärung von Bundeskanzler Willy Brandt war der DDR 1969 erstmals durch ein Mitglied einer Bundesregierung offiziell die Staatsqualität zuerkannt worden.* Der Weg zur internationalen Anerkennung der DDR und damit auch zur Aufnahme in die Vereinten Nationen sollte jedoch nach dem Willen der Bundesregierung an eine "akzeptable Formulierung der deutsch-deutschen Beziehungen zwischen den beiden deutschen Staaten geknüpft werden. ${ }^{14} \mathrm{Ob}$ wohl sich die DDR stets gegen diesen Sachzusammenhang ausgesprochen hat, konnte sich die Bundesrepublik mit ihrer Haltung durchsetzen. Erst mit der Paraphierung des Grundlagenvertrages am 8. November 1972 gab auch die Bundesrepublik grünes Licht für eine Aufnahme der DDR in internationale Organisationen. Mit der Aufnahme in die UNESCO am 24. November 1972 war der Bann gebrochen, da aufgrund der sog. "Wiener Formel" (Art. 48 des Wiener Abkommens über internationale Beziehungen) auch ein den Vereinten Nationen nicht angehörender Staat das Recht auf Aufnahme in alle Sonderorganisationen besitzt, sobald seine Aufnahme in eine der Sonderorganisationen erfolgt ist. ${ }^{15}$

Am 9. November 1972 veröffentlichten die Vier Mächte, USA, Großbritannien, Frankreich und die UdSSR, eine Erklärung, die Anträge auf

\footnotetext{
- Damit nicht verbunden ist die völkerrechtliche Anerkennung durch die Bundesrepubiik. Diese politische Position bedeutete gleichzeitig eine. Abkehr von der Identitätstheorie. Nach überwiegender westiicher Auffassung entspricht die Teilordnungslehre (zwei deutsche Teilordnungen mit eingeschränktem Völkerrechtsstatus unter dem handlungsunfähig fortbestehenden Deutschen Reich) der politischen Lage weit mehr.

13 Europa Archiv, 1969, S. D 500.

14 In Punkt 20 der Kasseler Punkte wurde festgehalten: "Die Bundesrepublik Deutschland und die Deutsche Demokratische Republik werden auf der Grundlage des zwischen ihnen zu vereinbarenden Vertrages die notwendigen Vorkehrungen treffen, um ihre Mitgliedschaft und Mitarbeit in internationalen Organisationen zu regeln." Materialien zum Bericht zur Lage der Nation, 1974, hrsgg. vom Bundesministerium für innerdeutsche Beziehungen; S. 6.

15 Siehe Bruns (Anm. 8), S. 33-37.
} 
Mitgliedschaft in der Organisation der Vereinten Nationen zu unterstützen, wenn sie von der Deutschen Demokratischen Republik und der Bundesrepublik Deuthschland gestellt werden. ${ }^{18}$ Am 21. Juni 1973 prüfte der Sicherheitsrat die Aufnahmeanträge der beiden deutschen Staaten und am 18. September 1973 wurden sie ohne formelle Abstimmung durch Akklamation als Mitglieder in die Weltorganisation aufgenommen.

Welche Konsequenzen hat die gemeinsame Mitgliedschaft der Bundesrepublik und der DDR für den Rechtsstatus der DDR aus der Sicht der Bundesrepublik? Verlieren mit der Aufnahme der beiden deutschen Staaten die Feindstaatenartikel ihre Gültigkeit? Welche Rolle spielte die Vier-Mächte-Verantwortung für Deutschland als Ganzes im Zusammenhang mit dem Beitritt in die Vereinten Nationen? Schließlich, wie ist die Vertretung Berlins in den Vereinten Nationen geregelt? Diese Sonderprobleme einer deutschen Mitgliedschaft sollen nachfolgnd kurz untersucht werden.

\section{Sonderprobleme einer deutschen Mitgliedschaft in den Vereinten Nationen}

\section{Die Anerkennung der DDR}

Hatte die CDU/CSU-Fraktion mit nur 4 Stimmen für den Grundlagenvertrag gestimmt, so waren es doch immerhin 99 Abgeordnete dieser Fraktion, die zusammen mit den Stimmen der SPD/FDP dem Aufnahmeantrag der Bundesrepublik in die Vereinten Nationen zustimmen. Der innenpolitische Konsens für diesen Schritt war damit erheblich breiter, jedoch nicht unumstritten. Welche Bedeutung kommt dem Beitritt der beiden deutschen Staaten in die Vereinten Nationen im Hinblick auf das Offenhalten der deutschen Frage und die völkerrechtliche Anerkennung der DDR zu?

"Das Völkerrecht kennt keine zwingenden Formvorschriften für die Anerkennung. Für die Rechtswirksamkeit des Anerkennungsaktes ist es daher unerheblich, ob der Wille, den neuen Staat als gleichberechtigtes Völkerrechtssubjekt zu respektieren, ausdrücklich ausgesprochen oder ob er durch konkludente Handlungen zum Ausdruck gebracht wird."17 $\mathrm{Zu}$ dem Problemgegenstand "Anerkennung durch Aufnahme in die Vereinten Nationen" lassen sich grundsätzlich drei Lehrmeinungen unterscheiden:

${ }_{16}$ Text abgedruckt in: Die Bundesrepublik Deutschland. Mitglied der Vereinten Nationen. Eine Dokumentation. 4., überarb. Aufl. Sankt Augustin 1981, S. 179. ${ }^{17}$ Gleich (Anm. 2), S. 40-41. 
1. Die gemeinsame Mitgliedschaft zweir Staaten ist gleichbedeutend mit einer konkludenten völkerrechtlichen Anerkennung.

2. Durch Aufnahme zweier Staaten, die sich gegenseitig nicht anerkannt haben, wird der Rechtsschein einer völkerrechtlichen Anerkennung erhoben. Dieser Rechtsschein kann jedoch durch die staatliche Willenserklärung eines Staates nichtig werden.

3. Die Aufnahme zweir Staaten beinhaltet, unabhängig vom Abstimmungsverhalten und von Vorbehalten keine konkludente völkerrechliche Anerkennung. ${ }^{18}$

Die vorherrschende Völkerrechtsmeinung stimmt darin überein, da $\beta$ die Úbernahme zur Pflicht der Förderung des gemeinsamen Beitritts zu den Vereinten Nationen keine konkludente völkerrechliche Anerkennung der DDR durch die Bundesrepublik darstellt.

Selbst wenn man in der Verpflichtung den gemeinsamen Beitritt zur UNO zu fördern, eine konkludente Anerkennung sähe, so könnte dies doch nur dann gelten, wenn die Bundesrepublik diese Verpflichtung vertraglich gegenüber der DDR übernommen hätte. ${ }^{19}$

Da die Bundesrepublik im Grundlagenvertrag das besondere rechtliche Verhältnis zwischen den beiden deutschen Staaten fixiert und damit die völkerrechtliche Anerkennung ausgeschlossen hat, ist auch das Argument des "Rechtsscheins" nicht gültig. Auch nach der Auffassung, daß eine gemeinsame Mitgliedschaft mit einer konkludenten Anerkennung gleichbedeutend ist, ist $\mathrm{zu}$ berücksichtigen, daß die Bundesrepublik zum Zeitpunkt der Aufnahme der DDR noch nicht Mitglied der Vereinten Nationen war, "sie hatte also ihre Anerkennungskompetenz (nach Kelsen) noch nicht auf die Organisation übertragen". ${ }^{20}$

\section{Die Feindstaatenartikel (Art. 53 und 107 der Charta der Vereinten Nationen)}

Mit der Aufnahme von Bundesrepublik und DDR sind alle ehemaligen "Feindstaaten" (Staaten, die während des Zweiten Weltkrieges Gegner eines Unterzeichners der Charta der Vereinten Nationen waren, Art. 53,2) Mitglieder der Vereinten Nationen. Allerdings ist umstritten, ob die Feindstaatenqualität von Territorium und Volk des ehemaligen Gegners ab-

$\overline{18 \text { Gleich (Anm. 2) }}$ S. 125-129; siehe auch: Dieter Blumenwitz, "Die beiden deutschen Stæaten in den Vereinten Nationen", in: Zeitschrift für Politik, 1977, S. 101-121. S. 102-107.

19 Gleich (Anm. 2), S. 133, Fußnnote 125.

${ }^{20}$ Gleich (Anm. 2), S. 132. 
hängt (Frage der Radiziertheit), sie sich also nicht auf die deutschen Staaten übertragen hat. ${ }^{21}$

Geht man jedoch davon aus, daß sich die Feindstaatenqualität auf die Bundesrepublik Deutschland und die DDR übertragen hat, so stellt sich nach dem Eintritt der beiden Staaten in die Vereinten Nationen die Frage nach dem Fortbestand der Feindstaatenartikel. Trützschler von Falkenstein kommt in seiner Untersuchung zu dem Ergebnis, da $\beta$ die westliche politische wie völkerrechtliche Argumentation davon ausgeht, da $\beta$ die Aufnahme in die Vereinten Nationen die Feindstaatenartikel gegenüber den Mitgliedern der Organisation gegenstandslos mache. Dies wird vor allem unter Bezug auf Art. 4,1 der Charta der Vereinten Nationen begründet: "friedliebend" (als Aufnahmebedingung festgehalten) und die Weitergeltung der Feindstaatenartikel gegenüber den ehemaligen Feindstaaten würden sich gegenseitig ausschließen.2 Die UdSSR schließt sich dieser Meinung jedoch nicht an, wie zuletzt aus der Erklärung Falins, des demaligen Botschafters der UdSSR in der Bundesrepublik, hervorgeht. ${ }^{23}$

Auch wenn den Feindstaatenartikel gegenwärtig keine politische Bedeutung zukommt, so ändert das nichts an der grundsätzlichen Fortdauer ihrer Gültigkeit-wenigstens solange wie Bemühungen um eine Revision der Charta bezüglich dieser Artikel nicht erfolgreich sind. Dies haben auch die beiden deutschen Staaten durch ihren Beitritt in die Vereinten Nationen anerkannt (Art. 4,1 der Charta der Vereinten Nationen).

\section{Die Vier-Mächte-Verantwortung für Gesamtdeutschland}

In der Erklärung vom 9. November 1972 haben die Vier Mächte ihr Einverständnis und ihre Unterstützung für die Mitgliedschaft von Bundesrepublik und DDR in den Vereinten Nationen erklärt und gleichzeitig festgehalten, da $\beta$ "diese Mitgliedschaft die Rechte und Verantwortlichkeiten der Vier Mächte und die bestehenden diesbezüglichen vierseitigen Regelungen, Beschlüsse und Praktiken in keiner Weise berührt." ${ }^{24}$

Die Rechte und Verantwortlichkeiten der Vier Mächte leiten sich her aus der bedingungslosen Kapitulation des Deutschen Reiches und den

2 Werner Trützschler von Falkenstein, Die sich ändernde Bedeutung der Feindstaatenartikel (Artikel 53 und 107 der Satzung der Vereinten Nationen) für Deutschland. Bern und Franüfurt/M. 1975 (Augsburger Schriften zum Staatsund Völkerrecht; 5), Kap. 4.

22 Trützschler von Falkenstein (Anm. 21), S. 100-101.

${ }^{23}$ Ebd.

24 Die Bundesrepublik Deutschland (Anm. 18). 
damit in Zusammenhang stehenden vertraglichen Abmachungen. ${ }^{25}$ Durch den Deutschland-Vertrag von 1954 und den Vertrag über die Beziehungen zwischen der DDR und der UdSSR vom 20. September 1955 sowie dem Freundschaftsvertrag zwischen diesen beiden'Staaten vom 12. Juni 1964 haben sich im Umfang der alliierten Rechte weitgehende Änderungen ergeben: weiterbestehende Souveränitätsbeschränkungen beziehen sich nur noch auf Deutschland als Ganzes und Berlin. Diese Souveränitätsbeschränkungen standen jedoch einem Eintritt der beiden deutschen Staaten in die Organisation der Vereinten Nationen nicht entgegen. Zum einen schließen sich Souveränitätsbeschränkungen und Aufnahme in die Vereinten Nationen nicht aus, zum anderen haben die Vier Mächte durch ihre Erklärung vom 9.11.1972 ihre Rechtsposition gewahrt. ${ }^{26}$

\section{Die Vertretung Berlins in den Vereinten Nationen}

In II D des Vier-Mächte-Abkommens vom 3. September 1971 in Verbindung mit Anlage IV wird die außenpolitische Vertretung der Berliner Westsektoren geregelt. ${ }^{27}$ Die Sowjetunion erkannte damit erstmals verbindlich die außenpolitische Vertretung der Interessen von Berlin (West) durch die Bundesrepublik Deutschland an. Die außenpolitische Vertretung der Interessen darf jedoch Gebiete betreffen, die Fragen der Sicherheit und des Status nicht berühren.

Der Begriff "Sicherheit" ist in diesem Zusammenhang als militärische Sicherheit zu verstehen; Angelegenheiten, die den Status betreffen, sind alle außenpolitischen Handlungen, durch die der Rechtsstatus Berlins, so wie er sich aus der bisherigén Entwicklung und aus dem Viermächte-Abkommen ergibt, unmittelbar geändert würde. ${ }^{28}$

In Nr. 2c der Anlage IV A und B wird der Bundesrepublik ausdrücklich das Recht zuerkannt, die Interessen von Berlin (West) in internationalen

25 Dokumente des geteilten Deutschland. Quellentexte zur Rechtslage des Deutschen Reiches, der Bundesrepublik Deutschland und der Deutschen Demokratischen Republik. Mit einer Einführung herausgegeben von Ingo von Münch. Stuttgart 1968, S. 25-29.

${ }^{26}$ Siehe dazu Wilhelm Kewenig, Sonderprobleme einer deutschen Mitgliedschaft in den Vereinten Nationen, in: Ulrich Scheuner und Beate Lindemenn, .Hrsg.. Die Vereinten Nationen und die Mitarbeit der Bunclesrepublik Deutschland. München und Wien 1973 (Schriften des Forschungsinstituts der Deutschen Gesollschaft für Auswärtige Politik e.V.; 32), S. 307-338, S. 322-323.

27 Europa Archiv, 1971 S. 450-451.

23 Ernst R. Zivier, Der Rechtsstatus des Landes Berlin. Eine Untersuchung nach jem Viermächte-Abkommen vom 3. September 1971. Berlin (West) 1973 (Völkerrecht und Politik; 8), S. 140. 
Organisationen und auf. internationalen Konferenzen zu vertreten. Dies wurde in dem Schreiben der Alliierten Kommandatura an den Regierenden Bürgermeister von B€rlin vom 13. April 1973 bekräftigt. ${ }^{29}$

Die Bundesrepublik hat ihrem Antrag an die Vereinten Nationen ein Schreiben beigefügt, in dem sie klarstellt, da $\beta$ sie "soweit nicht Fragen der Sicherheit und des Status berührt sind, die in der Charta der Verein-, ten Nationen enthaltenen Rechte und Verpflichtungen auch hinsichtlich von Berlin (West) übernehmen und die Interessen von Berlin (West) in den VN vertreten wird". ${ }^{30}$ Dieses Schreiben an den General-sekretär der Vereinten Nationen vom 13. Juni 1973 wurde als offizielles Dokument der Vereinten Nationen zirkuliert.

$\mathrm{Da} \beta$ die Sowjetunion mit der vertraglichen Festlegung der außenpolitischen Interessenvertretung durch die Bundesrepublik Deutschland keine generelle Billigung aller außenpolitischen Aktivitäten verband, sollte sich seither wiederholt erweisen. Dabei handelt es sich vornehmlich um die Auslegung der Begriffe "Sicherheit" und "Status". So wandten sich beispielsweise UdSSR und DDR gemeinsam gagen die Ausdehnung des Statuts der Internationalen Atomenergieorganisation und des Vertrages über die Nicht-Verbreitung von Kernwaffen auf Berlin (West) ${ }^{31}$

Der Ostsektor Berlins wird von der DDR vertreten, die, unterstützt von der Sowjetunion, diesen Teil Berlins als integralen Bestandteil der DDR betrachtet. Obwohl die Westmächte gegen diese Einbeziehung von Berlin (Ost) in die DDR protestieren und am Vier-Mächte-Status für ganz Berlin festhalten, haben sich die DDR und die. UdSSR zwar nicht rechtlich, aber politisch mit ihren Positionen durchgesetzt.

\section{Die Mitgliedschaft der beiden deutschen Staaten in den Vereinten Nationen}

Der folgende Abshnitt kann sich auf die wichtigsten Fakten der Mitgliedschaft von Bundesrepublik und DDR in den Vereinten Nationen beschränken, da eine Analyse der gegenwärtigen Politik in den Vereinten Nationen weniger völkerrechliche als politische Fragen und Probleme aufwirft. $\mathrm{Zu}$ untersuchen wäre in diesem Zusammenhang vor allem die

${ }^{29}$ Die Bundesrepublik (Anm. 16), S. 180.

${ }^{30}$ Ebd., S. 28-29.

31 Siehe hierzu Gerhard Wettig, Das Vier-Mächte-Azkommen in der Bewährungsprobe. Berlin im Spannungsfeld von Ost und West. Berlin (West) 1981 (Politologische Studien; 22), S. 193-196 und Wilhelm Bruns, Die Uneinigen in den Vereinten Nationen. Bundesrepublik Deutschland und DDR in der UNO. Köln 1980 (Bibliothek Wissenschaft und Politik; 25), S. 103. 
Politik der beiden deutschen Staaten in den Vereinten Nationen im Kontext ihrer Außenpolitik, Kooperations- und Konfrontations- muster, die sich durch diese Politik abzeichnen, Fragen der Formulierung ihrer Außenpolitik und Mittel ihrer Durchsetzung usw.

In den Jahren der beiderseitigen Mitgliedschaft hat die deutsche Frage im System der Vereinten Nationen keine Rolle gespielt und wird sicherlich in absehbarer Zukunft auch nicht spielen. Die Vertreter der beiden deutschen Staaten beschränken sich weitestgehend auf die Forumsfunktion der Generalversammlung, um ihre gegensätzlichen deutschlandpolitischen Standpunkte vorzutragen. ${ }^{32}$ Auch auf die Einbeziehung der deutsch-deutschen Problematik in der Behandlung der Menschenrechte im Rahmen der Vereinten Nationen soll hier nicht näher eingegangen werden. Hier sieht vor allem die CDU/CSU einen Wirkungsbereich, in dem die Mitgliedschaft der Bundesrepublik zu einer offensiveren Politik gegenüber der DDR genutzt werden soll. ${ }^{33}$

"Gleichberechtigt, aber ohne Gemeinsamkeiten", so überschreib Wilhelm Bruns eine Analyse der Politik der beiden deutschen Staaten in der 36. Generalversammlung der Vereinten Nationen. ${ }^{34}$ Diese Charakterisierung kann auch auf die gesamte Deuer der Mitgliedschaft von Bundesrepublik und DDR in den Vereinten Nationen stehen. Eine Abstimmung zwischen den beiden Staaten gibt es ebensowenig wie ein gemeinsames Abstimmungsverhalten. Im Gegenteil: Die Mitarbeit der Bundesrepublik Deutschland und der DDR im Rahmen der Vereinten Nationen verdeutlicht einmal mehr die Gegensätze in den (außen-) politischen Positionen. Dies zeigt sich besonders bei den vorherrschenden Themen der letzten Generalversammlungen: Internationale Sicherheit und Abrüstung (1. Ausschuß) und Entkolonisierung (4. Ausschuß). Während die Bundesregierung auf eine-auch hierzulande teilweise kritisierte-Politik des friedlichen Wandels in Südafrika setzt, unterstützt die offen die Befreiungsbewegungen, fordert umfassende Sanktionen des Sicherheitsrates gegen die Apartheidpolitik und schließt auch die Anwendung von Gewalt zur Lösung der Probleme im Südlichen Afrika nicht aus. ${ }^{35}$ Auch in Fragen der Abrüstung unterscheiden sich die Positionen der beiden Staaten grundsätzlich: so

32 Bruns (Anm. 31), S. 105-113.

33 Zur Gesamtproblematik siehe Blumenwitz (Anm. 18), S. 115-121. Zur Haltung der Bundesregierung Rüdiger Freiherr von Wechmar, "Die Bundesrepublik Deutschland in den Vereinten Nationen. Chancen und Grenzen unserer Mitwirkung", 1979, S. 113-120, S. 115.

34 Wilhelm Bruns, "Gleichberechtigt, aber ohne Gemeinsamkeiten. Die beiden deutschen Staaten auf der 36. Generalversammlung der UNO", in: Deutschland Archiv, 1982, S. 495-502.

35 Ebd., S. 199. 
"in der Beurteilung der Ursachen des Wettrüstens, in der Frage was prioritär verhandelt werden soll, und auf welcher Grundlage diese Verhandlungen vorangehen sollen". ${ }^{36} \mathrm{Da} \beta$ das Abstimmungsverhalten der DDR im Vergleich zu dem der Bundesrepublik dabei meist im Einvernehmen mit der Mehrzahl der Entwicklüngsländer steht, ist außer Frage.

Lä3t sich erwartungsgemäB eine nahezu 100\%ige Übereinstimmung des Abstimmungsverhaltens der DDR mit der UdSSR feststellen, so trifft diese Kongruenz des A.bstimmungsverhaltens der Bundesrepublik mit einem ihrer Hauptbündnispartner, den USA, sicher nicht zu: Abweichungen sind hier eher die Regel als die Ausnahme. ${ }^{37}$.

Die gleichberechtigte Mitgliedschaft der DDR mit der Bundesrepublik - zunächts in den Sonderorganisationen und später auch in den Hauptorganen der UNO selbst - war erklärtes Ziel der Führung der DDR in den 50er und 60er Jahren. Mit der Aufnahme in die UNESCO 1972 und dem gemeinsamen Beitritt in die Vereinten Nationen hat sie diese gleichberechtigte Mitgliedschaft erreicht. In Organisationen, in denen die DDR bis heute nicht vertreten ist, ist dies auf Gründe zurückzuführen, die nicht immer eindeutig und überzeugend sind und die der propagierten aktiven uneingeschränkten Mitgliedschaft in der Organisation der Vereinten Nationen widersprechen. Diese Politik der selektiven Mitgliedschaft bezieht. sich in erster Linie auf die Mitgliedschaft in den Sonderorganisationen (siehe Anlage I). Relativ eindeutig ist der Sachverhalt der Nichtmitgliedschaft der DDR in der sog. Weltbankgruppe (IBRD, IFC, IMF, IFAD), die als Instrument imperialistischer aggressiver Kapitalexpansion und des Neokolonialismus dargestellt wird. ${ }^{38}$ Die DDR folgt in diesem Falle. ebenso wie bei der FAO dem Vorbild UdSSR, die diesen Sonderorganisationen ebenfalls fernbleibt. Nicht zutreffend ist diese Begründung jedoch im Falle der ICAO. Hier ist die DDR der einzige kommunistische Staat Mittel- und Osteuropas der nicht Mitglied ist.

Als Grundregel für die Mitgliedschaft kommunistisch regierter Staaten in Sonderorganisationen darf die Außerung von W. Morawiecki ${ }^{33}$ gelten, nach der die Ziele der Sonderorganisationen diesen Staaten nicht "fremd" oder "feindlich" sein dürfen und sie von Anfang an "gleichberechtigt" an der Gründung der Sonderorganisationen beteiligt gewesen sein müssen. Eine solche Begründung ist jedoch schwer faßbar und für. vielfältige Interpretationen offen.

\footnotetext{
36 E.bd., S. 497; siehe auch S. 502.

${ }^{37}$ Bruns, Die Uneinigen (Anm. 31), S. 62.

Kleines Politisches Wörterbuch. 3., überarb. Aufl. Berlin (Ost) 1978, S. 661-662. Bruns, Politik (Anm. 10), S. 155-156.

32 Zitiert in Bruns, Politik (Anm. 10), S. 155.
} 
Die Zeit der Mitgliedschaft der Bundesrepublik Deutschland und der DDR in den Vereinten Nationen, in der jeder der beiden Staaten von seiner Regionalgruppe auch bereits als Vertreter in den Sicherheitsrat gewählt worden ist, ${ }^{40}$ hat gezeigt, daß diese die deutsche Frage - erwartungsgemä $\beta$ nicht weitergebracht, aber die deutsche Teilung auch nicht weiter vertieft hat. Die Tätigkeit der beiden deutschen Staaten wird gemessen an ihren politischen Aktivitäten und ihren materiell-finanziellen Beiträgen. Unter diesen Gesichtspunkten sollte auch die Bundesrepublik die Politik der DDR in der Weltorganisation beurteilen und umsetzen.

10 Siehe hierzu auch Bruns, Die Uneinigen (Anm. 31). S 148 